\title{
Total Quality Management [TQM] in the Healthcare Industry - Challenges, Barriers and Implementation Developing a Framework for TQM Implementation in a Healthcare Setup
}

\author{
M. Balasubramanian \\ Hinduja Healthcare Surgical, Medical Superintendent, Department of Hospital Administration, Mumbai, India \\ Email address: \\ mbalas2001@hotmail.com
}

\section{To cite this article:}

M. Balasubramanian. Total Quality Management [TQM] in the Healthcare Industry - Challenges, Barriers and Implementation Developing a Framework for TQM Implementation in a Healthcare Setup. Science Journal of Public Health. Vol. 4, No. 4, 2016, pp. $271-278$. doi: $10.11648 /$ j.sjph.20160404.11

Received: February 26, 2016; Accepted: May 17, 2016; Published: June 2, 2016

\begin{abstract}
Quality management has become an important issue in healthcare organizations (hospitals) during the last couple of decades. The increased attention to quality is due to governmental regulations, influence of customers, and hospital management initiatives. Apparently, there are many difficulties in managing healthcare organizations in a competitive marketplace with a little support from official bodies especially in a developing country like India. The purpose of this paper is to provide a framework for implementing the total quality management concept that is compatible with the local culture of India. The SERVQUAL model and its application can help the healthcare facility in achieving satisfaction on both ends employees' satisfaction and customer satisfaction. In healthcare organizations, the traditional Indian culture, leadership style, and the mentality of the medical professionals are somehow the barriers to the adoption of the TQM. The suggested integrated framework model of the TQM can be of great help to the healthcare organizations to move out of the barriers and successfully implementing TQM concepts and practices.
\end{abstract}

Keywords: TQM: Total Quality Management, SERVQUAL: Service Quality, QFD: Quality Function Deployment

\section{Introduction}

Quality management has become an important issue in healthcare organizations (hospitals) during the last couple of decades. The increased attention to quality is due to governmental regulations, influence of customers, and hospital management initiatives. So, the role of government as the main provider of healthcare (HC) services has changed. Additionally, the healthcare market is changing from a producer-oriented to a customer-oriented market due to the increasing influence of customers and public pressures. As a consequence, the patient is becoming a customer for the healthcare organizations, or more likely a direct strategic partner who participates in a decision making process. The changes in environment, society, and political policies have significant impacts on management in hospitals as well. There are many difficulties in managing healthcare organizations in a competitive marketplace with a little support from official bodies especially in a developing country like India. The evolution of concepts such as TQM and Six Sigma has only added to the repertoire of the word. TQM 'Total Quality Management' is practised widely at different organizations and Hospitals are no exception to this.

The Academicians and HR practitioners have discussed about TQM 'Total Quality Management' as a new concept that had its roots in the Japanese management style. Zandin (2001) mentions that TQM helps improve the quality of services and goods through a collaborative approach and standardized performance. Whyte and Witcher (1992) explain TQM as an approach with a holistic perspective on Total, Quality and Management. Total, because TQM takes input from every department and individual; Quality, because TQM helps attain standard on customer service and end-user satisfaction; Management, because TQM brings to fore innovative new forms and practices on Management. The researchers do seem to explain Total Quality Management as a management philosophy to achieve Excellence on Business. 
The purpose of this paper is to provide a framework for implementing the total quality management concept that is compatible with the local culture of India. The study verifies that the proposed framework model provides an unbiased, perspective and comprehensive view of hospital reality that can human and other resources, patients, activities to organizational units

\section{TQM - Definition for Healthcare}

In healthcare services there are three definitions distinguished TQM from other approaches:

"TQM is a comprehensive strategy of organizational and attitude change for enabling personnel to learn and use quality methods, in order to reduce costs and meet the requirements of patients and other customers" (Ovretveit, 2000)

"Maximization of patient's satisfaction considering all profits and losses to be faced in a healthcare procedure" (Donabedian, 1989).

TQM is a management method: "TQM/CQI - Continuous Quality Improvement - is about two things: a management philosophy and a management method". They propose four "distinguishing functions", which are often defined as the essence of good management which includes:

- Empowering clinicians and managers to analyze and improve process;

- Adopting a norm that customer preferences are the primary determinants of quality and the term "customer" includes both the patients and providers in the process;

- Developing a multidisciplinary approach which goes beyond conventional departmental and professional lines; and

- Providing motivation for a rational data-based cooperative approach to process analysis and change

\section{Theoretical Background}

Laffel \& Blumenthal, 1989 define TQM in a healthcare setup -

1- An active, visible support from clinical and managerial leadership for continuous quality improvement.

2- A focus on processes as the objects of improvement.

3- The elimination of unnecessary variation.

4- Revised strategies for personnel management.

Thomas Plochg \& Niek Klazing (2005)

By inference from this analysis, a twofold agenda for a constructive dialogue is proposed. Doctors and managers must develop a shared vision of the division and coordination of medical work as well as discussing the values, norms and goals underlying patient care. It's a questionable, however, whether this agenda is currently adequately addressed.

Ching Horng and Fenghuhei Huarng (2001) conducted a deep study by collecting data from 76 hospitals in Taiwan to test a multilevel model addressing the issue of TQM adoption as one type of organizational adoption.
They focused on the extent of TQM adoption by the individual hospitals as dependent variable. As for the independent variables, they selected five multilevel constructs: the scope of the network cooperation, the nature of the network relationship, organizational identity, adoption strategy and organizational citizenship behavior.

Results from the regression analysis indicate that both the nature of the network relationship and prospector strategy is positively and significantly related to the extent of TQM adoption

Theodorakioglou, D (2000) conducted a research in public hospitals in 1998. The research analysis showed that the hospital sector is facing a serious crisis. The main conclusions of the research were as follows:

- The implementation of quality management systems in public hospitals is rare. In most cases, the successful implementation of quality programs is excluded, since an active and visible support from managerial leadership does not exist.

- The lack of information systems for data saving and filing obstructs the effective diffusion and use of information.

- The ignorance of essential quality terms by superior managers and physicians is discouraging but also indicative of the lack of information and appropriate education of hospital employees regarding quality issues.

- Owing the insufficiency of hospital staff, operational problems are apparent in hospitals.

i. Accountability:

Total quality management is a system that makes quality the responsibility of all clinicians and administrators throughout the health care organization.

ii. Alleviating Problems:

In TQM, systems are established to prevent clinical and administrative problems, increase patient satisfaction, continuously improve the organization's processes, and provide healthcare services as good, or better, then those of the competitors.

iii. Raising Fundamental Metrics:

Customer focus, error prevention, employee participation, teamwork, systemization, leadership and continuous quality improvement are TQM universal management concepts that can be transferred to any business setting. TQM uses quality as the fundamental measurement metric, continuous improvement as the philosophy and employee involvement as the approach.

iv. Measurable:

TQM programs in healthcare can be measured, without the need for a deep knowledge of the industry unique circumstances

\section{Defining Quality for Patient Care}

The term quality of patient care is still subject to debate and has no specific definition yet, since the guides to quality of healthcare are highly probabilistic due to estimations of 
the outcomes. The outcomes are not certain; therefore the measures of its quality must include dimensions of both the outcome of the care provided and processes by which the care is carried out. The measures of quality of care should include an evaluation of the provider-patient relationship.

For the purpose of this study the definition of quality of healthcare has been developed to consider the patient's, the doctor's, and other supporting factors perspectives. Hence, the following definition was used to encounter all above mentioned standpoints. Healthcare is "the proper implementation of an agreement between a patient, a physician, a nurse, and/or physicians and a healthcare organization regarding a medical intervention that is in a harmony with actual professional standards and protocols applied within the organization".

Specifying QUALITY

1- Fundamentals of care:

The basic level at which quality principles will apply across all areas of care, characteristics at this level may include, privacy, communication, responsiveness, and empathy.

2- Generate area of care:

Those areas which are common to a limited range of specialties or conditions, such as cancer treatment services, day case surgery, and emergency care. Quality characteristics at this level would include acceptability, information, appropriateness and equity.

3- Clinical specialty:

This level allows principles to apply to all patients cared for by a specialty area such as physiotherapy, radiology; quality characteristics in this level would include accessibility, efficiency, and reliability.

4- Individual condition or care group:

At this level, care is considered for particular conditions or patient care group, such as diabetes, maternity. Quality characteristics would focus on technical issues an example of clinical appropriateness and effectiveness.

The quality is assessed in terms of

- Quality of Technical Care

- Quality of the art of Care

- Quality of the Environment

These appraisals are done with reference to three variables.

- Structural Variables

- Minimum infrastructure required for making available a particular service

- Clarity regarding

- Buildings

- Space

- Equipment

- Environment

- Staff

\section{Model on TQM - Deming Model}

Deming, in his model, defined TQM as a method of leadership and management which:

- Analyzes system for errors and variation rather than blaming people;

- Develops long term partnership with external and internal suppliers;

- Uses accurate data to analyze processes and measure system improvement;

- Involves the staff who work in system analysis and improvement;

- Sets up effective collaborative meetings as the basis of teamwork;

- Train supervisors and managers in leading the ongoing improvement process;

- Engages staff in setting targets and ensures that results are feedback;

- Highlights the need for senior executives to plan strategically;

- Achieves long-term improvement through small incremental steps

\section{Measuring Service Quality}

Three characteristics of healthcare services contribute to the difficulty of measuring it, these are:

- Service intangibility

- Performance heterogeneity

- Customer-producer inseparability

However, unlike other services in the healthcare context, the patient/customer participates in the delivery of the service, and therefore, both performance and quality will be affected by the patient/customer actions, moods, and cooperativeness.

These dimensions of health care services make it:

- More difficult for customer/patients to evaluate

- Evaluations made are not only on the output but also on the delivery process itself

Parasuraman (1991) shown the conceptualization of the customer's evaluation of overall service quality as the gap between expectations and perceptions of service performance levels. Furthermore, he proposed the SERVQUAL tool, which was designed to measure service quality.

\section{SERVQUAL Model}

In healthcare patient satisfaction is the aim of using SERVQUAL which will be used later in this study to assess and measure the patient's satisfaction. It is important to note here that patient satisfaction affects a number of vital issues. Since it affects the use of the medical service provided by healthcare organizations, it also affects the kind of relationship between the patient and service providers. Moreover, patient satisfaction data contribute to the monitoring of healthcare delivery at all levels within the organization. i.e.

- Organizational level (hospital, clinic, etc.)

- Unit level (surgical, laboratory, etc.)

- Individual level (nurses, physicians, etc.)

Patient satisfaction is a patient's (affective or emotional) response to his or her (cognitive or knowledge-based) 
evaluation of the healthcare provider's performance (perceived quality) during a healthcare consumption experience.

I. Patient satisfaction inquiries are very popular in healthcare due to several reasons behind this, the efforts of quality management and improvement, to increase attention to customers. Therefore, patient satisfaction has been seen as a measure of quality,

II. Questionnaires are the most commonly used instruments for data collection. It is increasingly recognized that patients should be given a voice in the assessment of the service quality that is offered to them by the healthcare organizations.

The well known SERVQUAL tool is considered as a good approach for measuring the customer satisfaction. Patient satisfaction surveys are often seen as the natural outcome of the increase in consumerism. The SERVQUAL methodology is primary developed to measure satisfaction with service industries. The approach starts with the hypothesis that service quality is critically determined by the difference between customers' expectations and perceptions of the services. The method is predicated upon the gap to be discerned between customers' expectations of a service and their perceptions of service as actually experienced.

Research by (Parasurman, et. al., 1991) has shown that regardless of the type of service, customers use basically similar criteria in evaluating service quality. The criteria fall into ten key categories as follows:

- Reliability, which involves consistency of performance and dependability.

- Responsiveness, concerns the willingness or readiness of employees to provide service. It involves timeliness of service.

- Competence means possession of the required skills and knowledge to perform the service.

- Access involves approachability and ease of contact.

- Courtesy involves politeness, respect, consideration and friendliness of contact personnel.

- Communication means keeping customers informed in language they can understand and listening to them.

- Credibility involves trustworthiness, believability and honesty. It involves having the customer's best interest at heart.

- Security is the freedom from danger, risk or doubt.

- Understanding involves the efforts to understand the customer's needs.

The gap between expectations and perceptions may be analyzed with respect to five dimensions determined from an examination of the content of the ten service quality items discussed earlier. The final list of dimensions and their concise definitions will be as follows:

1. Tangibility: physical facilities, equipment and appearance of personnel.

2. Reliability: ability to perform the promised service dependably and accurately.

3. Responsiveness: willingness to help customers and provide prompt service.
4. Assurance: knowledge and courtesy of employees and their ability to inspire trust and confidence.

5. Empathy: caring, individualized attention the firm provides to its customers.

Application of SERVQUAL can be used to make comparisons globally over time. It's possible to ascertain those elements of service in which the gap between expectations and perceptions is widest. The application of this instrument and results of measurement allows possibilities of more specific management action to readdress perceived outcomes.

\section{SERVQUAL - Barriers}

- Limited Commitment: The successful introduction of TQM in hospitals requires the involvement and commitment of all parties in providing the service. Yet, this commitment presents a problem for hospitals since not all the parties are directly employed by these hospitals. The physicians and specialists usually work on their own and have limited commitments with the hospital they corporate with.

- Diffusion of Interests: Most doctors believe that their interests are not directly tied to that of the hospitals they work with. In fact, barriers by physicians' involvement may turn out to be the most important single issue impending the success of quality improvement in medical care.

- Structure of the Hospital: The difficulty of involving physicians in TQM has its roots in hospital structure. In most hospitals usually two bodies exist; the hospital on one side and the medical staff on the other side with all the conflicts between these two bodies.

Apprehensions with SERVQUAL

"Doctors view patient care as very individual and private affairs. As members of an old and highly respected profession, physicians have operated autonomously and have been accountable only to themselves and their peers"

"The complexity of medical care kept them immune from outside scrutiny and regulation. Because of these privileges physicians perceive themselves as losing power and influence, and may see TQM as an assault on their independence"

"The doctors view TQM as a program which will replace what was largely subjective process controlled by them, with as objective and statistically based discipline which is not under their control"

- Physician's Resistance: The tools and methods of TQM are foreign to physicians and considered abstract and irrelevant to individual patient care. Therefore, finding a way to overcome physicians' resistance becomes a priority for the managers who are seeking a successful and fruitful implementation of TQM in hospitals.

- Avoiding Fear: It is very important that doctors become committed to quality. This can be achieved by avoiding a threatening presentation for the TQM program, building common believes among physicians by 
involving them from the early stages of TQM this involvement will increase the chances of success.

- Begin with the Loyalists: Lopresti and Whetstone (1993) recommended that involving physicians in establishing TQM should begin with a few interested physicians and expand to others as their involvement begins to attract support. Thus, to resolve the conflict between doctors and hospitals a new leadership style is again needed a one that is capable of bridging the between the two bodies

Table 8.1. Professional vs TQM views.

\begin{tabular}{lll}
\hline Area of Conflict & Professional view & TQM View \\
\hline Responsibility & Individual & Collective \\
Leadership & Individual & Managerial \\
Autonomy & Individual / Peers & Accountability \\
Planning & Rigid & Flexible \\
Feedback & Responses to complaints & Benchmarking \\
Performance Appraisal & Retrospective & Continuous \\
Authority & Administrative & Participative \\
\hline
\end{tabular}

- Lack of resources

Insufficient or improper infrastructure, equipment, manpower make it impossible for output of the desired quality.
- Drugs and Medical Supplies

Non- availability of essential drugs and supplies, spurious, adulterated drug preparation have a deleterious effect on the course of hospitalization and final prognosis.

\section{- Improper Maintenance}

To minimize equipment downtime, it is necessary to ensure adequate after-sales service.

\section{- Personnel Problems}

Lack of adequately trained, skilled and motivated employees compromise on the quality of care.

- Unreasonable patients and attendants

Illness, anxiety, etc cause patients, their families and friends to adopt unreasonable and uncooperative attitudes.

\section{Problem Statement}

As discussed, the tertiary care hospitals are at present facing problem on different accounts. The medicinal prices are rising with each day, infirmaries are in a shabby state, the mortality rates are on the rise and healthcare facilities are in a sorry state. This is further substantiated by Crisil's present statistics on medical infrastructure in India.

\section{India Health Infrastructure}

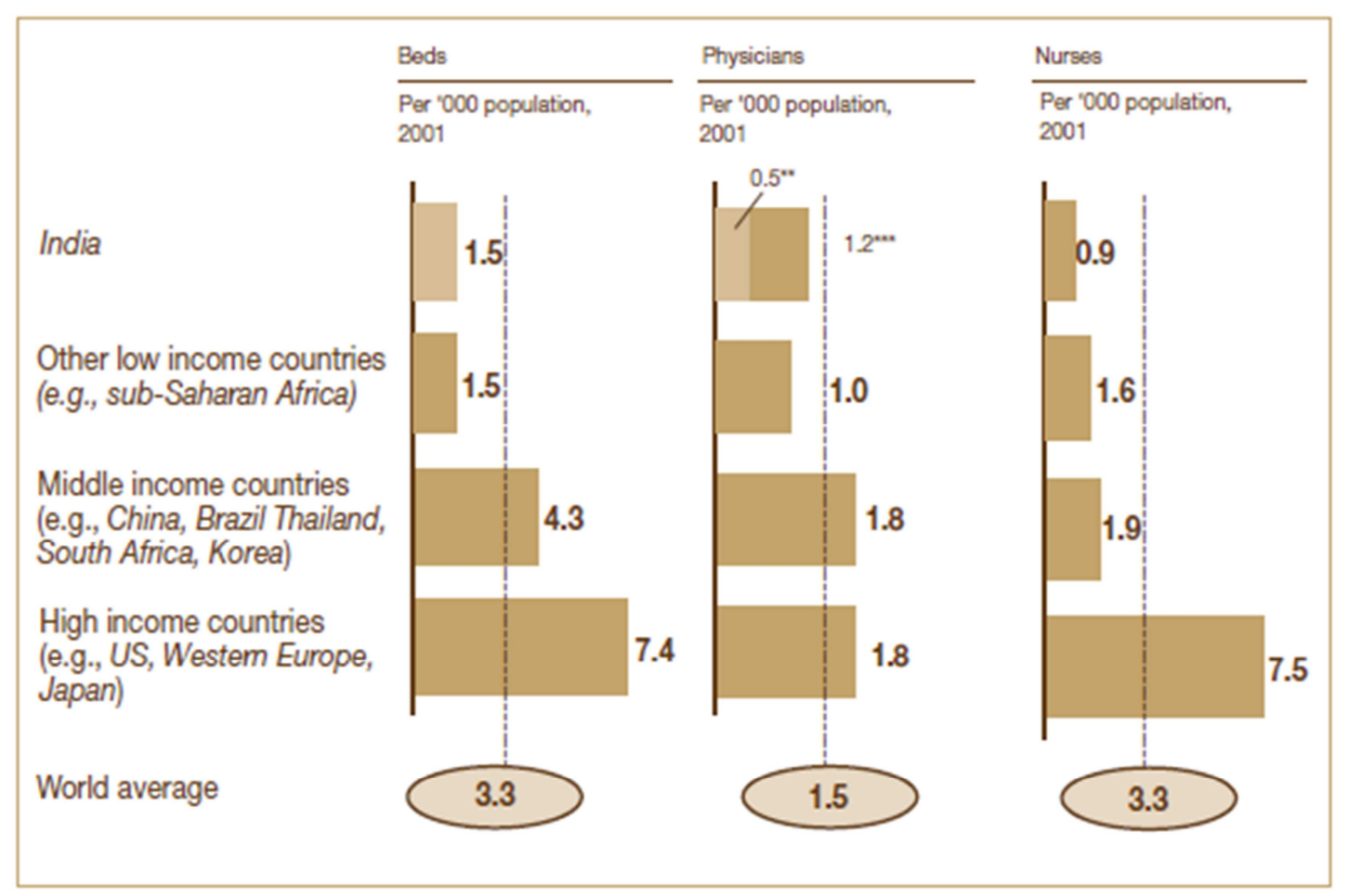

Fig. 9.1. India Health Infrastructure.

Against a world average of 3.3 beds, India has only 1.5; against a physician density of 1.5 , India has only 0.5 and against a nurse density of 3.3 , India has only 0.9 . Even the Middle Income countries like Brazil, Thailand and China score good over India. Clearly, 'quality' is still a distant dream for hospitals in India. The country has a population of 1.4 billion and this gets complicated without an infrastructure on medical insurance. Also, the 'cost' on acquiring technology and medical infrastructure is rising with each day.

There is a loud and clear message that TQM is not easy to implement in India. Even if one wants to do this the challenges are huge. This study therefore goes beyond the definition of the term TQM 'Total Quality Management' to identify challenges in its application and a likeable remedy to this. 


\section{SERVQUAL - Implementation}

A planned programme- monitors \& evaluates clinical performances of all practitioners

- Identifies opportunities for improvement

- Provides a mechanism for sustainable improvements

The framework development will follow the below sequence:

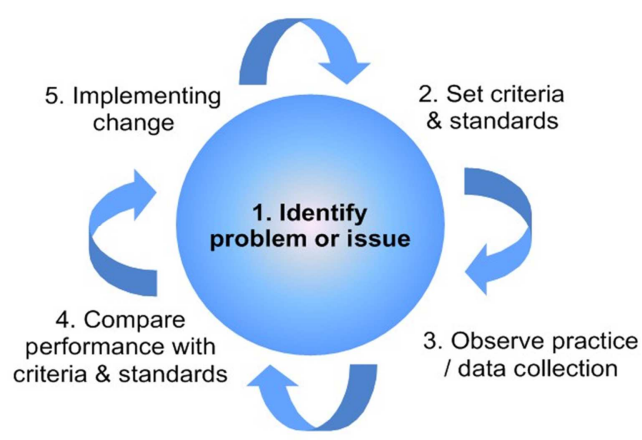

Fig 10.1. Servqual Framework.

1- Establishing commitment for the change. Top management of the healthcare organization must trust in the success of TQM implementation. This trust must be transferred to the employees by explaining the reasons behind the adoption of TQM.

2- Settings the principles and policies of quality. The first thing or action to do for implementing TQM successfully is to start changing the traditional organizational culture to become quality oriented and customer oriented. This change must be managed properly by the top management that has to start listening to the internal customers, participate in teams, in other words the top management must start to show practical support to TQM programs.

3- Establishing the right infrastructure for the change. The top management and its partners in the change must try to correctively instil new concepts of quality by making quality the core of meetings, communications, the new mission, vision, and goal statement, must all evolve around the new concept and how it has to be carried out.

Five methods of monitoring are as follows:

- The variation from the norm in the outcome

- Departure from specified outcome criteria

- Comparison of small groups in the same field

- Surveys

- Peer-review

Four important principles for effective implementation are...

- Attention should be focused on aspects of care

- Common conditions should be handled first

- Medical audit must be kept simple

It must be ensured that task undertaken are achievable and are achieved

$i$. Conducting educational training.

To make quality a way of living and a trend of thinking employees must be subject to intensive training programs that should aim at the education of the participants.

\section{ii. Constructing the teamwork.}

For TQM to fully function teamwork and participation are the engineering powers, especially in the medical service.

iii.Adjusting the style of leadership.

The top management should start by now to move on from the old fashion management style - control style - to a more open style based on encouragement listening, teamwork style - innovative style.

Key-steps

- Knowing the internal customers' requirements. Now and after the fact that all employees are familiar with the new concept, it is time to understand their requirements.

- Knowing the external customers' requirements. TQM has a wonderful tool in which customers' requirements may be detected. Quality Function Deployment (QFD) is usually used in order to clarify the customers' needs, requirements, and expectations in order to take action to satisfy these needs.

- Standardization for managing the change. It is very important for clinical path to become standardized in order to assure the quality of the service - medical treatment - and to save effort, money and time.

- Carrying on with continuous improvement. Based on the results of using QFD, management must make continuous improvements to keep its customers satisfied.

- Performing customer satisfaction surveys. It is very important to conduct a customer satisfaction survey periodically to analyze the problems that continue to persist, in order to take further action.

- Spreading the right quality concept. A change of culture is now in place and it is about time for the employees to accept the new trend and start to act accordingly.

\section{Conclusion}

The SERVQUAL model and its application can help the healthcare facility in achieving satisfaction on both ends employees' satisfaction and customer satisfaction. In healthcare organizations, the traditional Indian culture, leadership style, and the mentality of the medical professionals are somehow the barriers to the adoption of the TQM. The suggested integrated framework model of the TQM can be of great help to the healthcare organizations to move out of the barriers and successfully implementing TQM concepts and practices. It is therefore important to verify that the proposed framework model provides an unbiased, perspective and comprehensive view of hospital reality that can link human and other resources, patients, activities to organizational units. A view that will help hospitals to avoid falsifiability which may take place through two ways:

- The focus on only a part of the hospital performance which is the successful one,

- The use of a biased view of performance, for example certain departments deal with patients with severe medical conditions (Cancer) leading to an apparently lower performance level. 


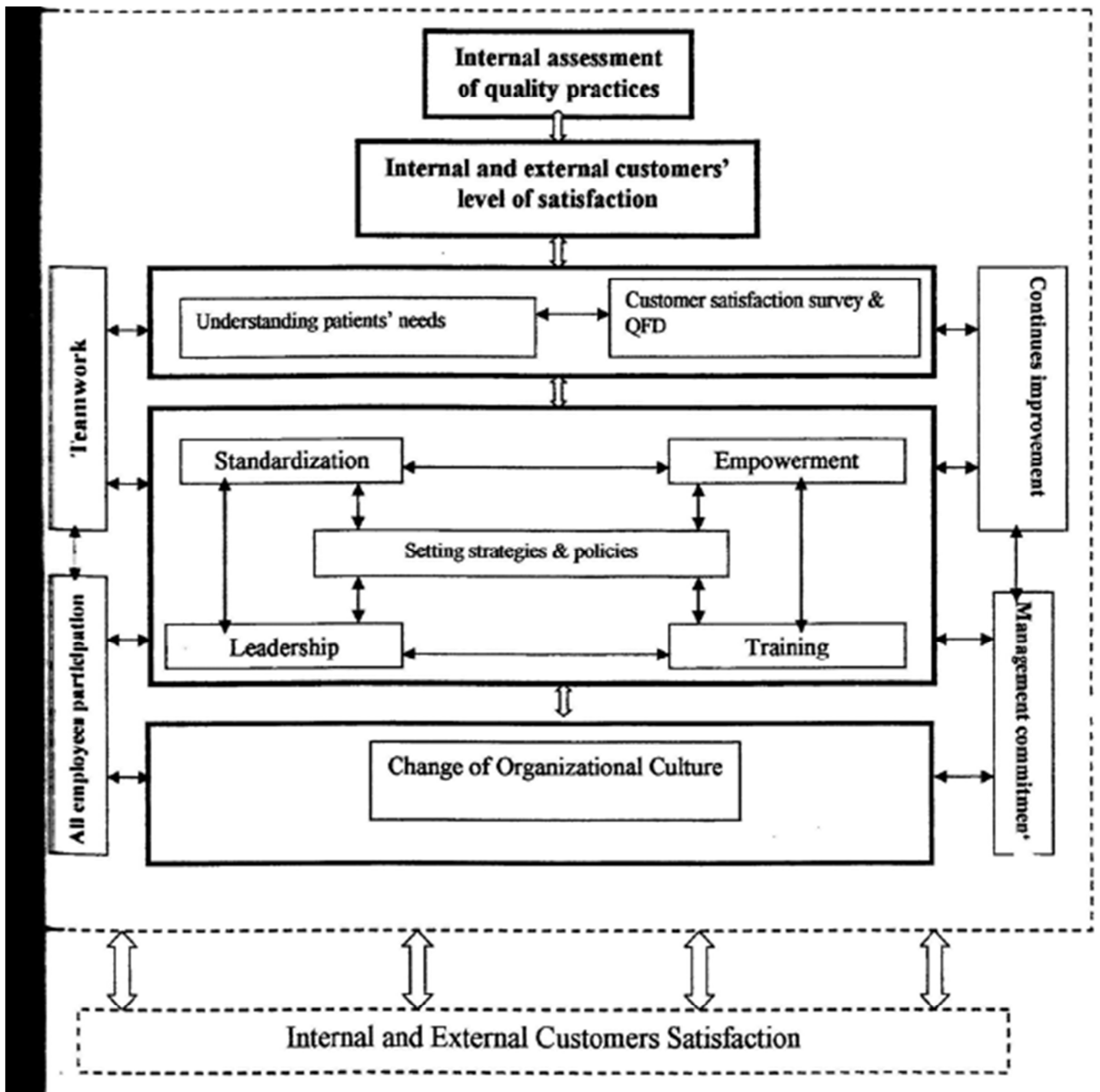

Fig. 11.1. Tqm implemetation-framework model.

\section{References}

[1] Alsharif B. F. T. 2008. Patient's satisfaction with hospital services at Nablus district, west bank, Palestine. Unpublished master thesis.

[2] Badri M. A., Attia S. and Ustadi A. M. 2009. Healthcare quality and moderators of patient satisfaction: testing for causality. International Journal of health care quality assurance.

[3] Ching H., and F. Huarng (2002), "TQM adaption by hospitals in Taiwan", Journal of Total Quality Management., Vol. 13, No. 4, 2002.

[4] Conway, T. and S. Willcocks, (1997), "The role of expectations in healthcare quality", International Journal of Healthcare Quality, Vol. 10, No. 3, 131-140.

[5] Donabedian, A, (1989), "Institutional and professional responsibilities in quality assurance", Quality Assurance in Healthcare, Vol. 1, 3-12.
[6] Gorst, C. et. al. (1998), "Psychological squeal of torture and organized violence suffered by refugees from Iraq. Traumarelated factors compared with social factors in exile", The British Journal of Psychiatry, 172: 90-94.

[7] Kovner A. R. and Knickman J. R. 2008. Health care delivery in the United States, $9^{\text {th }}$ edition, Springer Publishing Company. New York.

[8] Laffel G, and. Blumenthal, (1989), "The case for using industrial quality management science in health care organizations”, JAMA, Nov 24; 262 (20): 2869-73.

[9] Larrabee, J. H, and L. V. Bolden, (2001), "Defining patients perceived quality of nursing care", Journal of Nursing Care Quality, Vol. 16, No. 1, pp. 65-75.

[10] Lopresti, J, et. al. (1993), "Total Quality Management: Doing Things Right, Nursing Management", January, Volume 24 Issue 1, 7-76.

[11] Lynn, M. R. et. al, "2007", Understanding and measuring patients 'assessment of the quality of nursing care", Nursing Research, May/June, Vol. 56, No. 3, 159-166. 
[12] Naidu A. 2008. Factors affecting patient satisfaction and health care quality. International Journal of health care quality assurance

[13] Newman, K., (2001), "The nurse retention, quality of care and patient satisfaction", International Journal of Healthcare Quality Assurance, Vol. 4, No. 2, 57-68.

[14] Overtveit, J., (2000), "Total quality management in European healthcare", International Journal of Healthcare Quality Assurance, Vol. 13, No. 2, 74-90.

[15] Overtveit, J., (1996), "medical participation on a leadership of quality program", Journal of Management in Medicine, Vol. 10 , No. $5,21-28$.

[16] Parasurman, A, (1991), "SERVQUAL: a multiple-item scale for measuring customer perceptions of service quality", Journal of Retailing, Vol. 67.
[17] Parasurman, A, and et. al., (1991), "Understanding customer expectations of service”, Sloan Management Review, Spring.

[18] Plochg, T, et. al, (2005), "Intermediate care: for better or worse? Process evaluation of an intermediate care model between a university hospital and a residential home", BMC Health Service Res., May 24; 5: 38.

[19] William, A. S, and J. K. Johnson, (2013), "Mclaughlin and Kaluzny's continuous quality improvement in healthcare", 4th.ed. Jones \& Bartlett Learning.

[20] Whyte, J., \& Witcher, B. (1992). The Adoption of Total Quality Management in Northern England. Durham: Durham University Business School.

[21] Zandin, K. B. (2001). Maynard's Industrial Engineering Handbook. Fifth Edition. New York, NY: McGraw-Hill. 